submitted to Geophysical Prospecting.

\title{
Full waveform inversion using oriented time-domain imaging method for vertical transverse isotropic media
}

\author{
Zhen-dong Zhang and Tariq Alkhalifah \\ Department of Physical Science and Engineering, \\ King Abdullah University of Science and Technology, \\ Thuwal 23955-6900, Saudi Arabia. \\ Emails: zhendong.zhang@kaust.edu.sa8j tariq.alkhalifah@kaust.edu.sa
}

This article has been accepted for publication and undergone full peer review but has not been through the copyediting, typesetting, pagination and proofreading process, which may lead to differences between this version and the Version of Record. Please cite this article as doi: 10.1111/1365-2478.12560. 


\section{ABSTRACT}

Full waveform inversion for reflection events is limited by its linearized update requirements given by a process equivalent to migration. Unless the background velocity model is reasonably accurate, the resulting gradient can have an inaccurate update direction leading the inversion to converge what we refer to as local minima of the objective function. In our approach, we consider mild lateral variation in the model, and thus, use a gradient given by the oriented time-domain imaging method. Specifically, we apply the oriented time-domain imaging on the data residual to obtain the geometrical features of the velocity perturbation. After updating the model in the time domain, we convert the perturbation from the time domain to depth using the average velocity. Considering density is constant, we can expand the conventional 1D impedance inversion method to $2 \mathrm{D}$ or $3 \mathrm{D}$ velocity inversion within the process of full waveform inversion. This method is not only capable of inverting for velocity, but it is also capable of retrieving anisotropic parameters relying on linearized representations of the reflection response. To eliminate the cross-talk artifacts between different parameters, we utilize what we consider being an optimal parametrization for this step. To do so, we extend the prestack time-domain migration image in incident angle dimension to incorporate angular dependence needed by the multiparameter inversion. For simple models, this approach provides an efficient and stable way to do full waveform inversion or modified seismic inversion and makes the anisotropic inversion more practicable. The proposed method still needs kinematically accurate initial models since it only recovers the high-wavenumber part as conventional full waveform inversion method does. Results on synthetic data of isotropic and anisotropic cases illustrate the benefits and limitations of this method.

Key words: Anisotropy, Full waveform, Inverse problem 


\section{INTRODUCTION}

Full waveform inversion (FWI) in its inception was meant to utilize all the information in waveforms to reconstruct subsurface models responsible for the distinctive make-up of recorded data. The common practice of implementation of FWI includes a least-squares local optimization, in which the velocity perturbation is estimated from the data residual to update the background velocity model (Virieux \& Operto 2009). Lailly (1983) and Tarantola (1984) have shown that such perturbations are given by cross-correlating the forward-propagated source wavefield and the back-propagated data residual. However, the data residual can be cycleskipping due to the inherent oscillation property of seismic waves. To avoid being trapped into local minima, the conventional FWI method needs a good initial model as an input. Usually, the tomography methods such as wave-equation traveltime tomography (WT) and migration velocity analysis (MVA) can provide a good initial model for the conventional FWI method (Luo \& Schuster 1991; Liu et al. 2010; Weibull et al. 2012; Feng \& Schuster 2016). Alternatively, the global optimization method (Sen \& Stoffa 1991; Datta et al. 2016), anti cycle-skipping objective functions (Van Leeuwen \& Mulder 2010; Bozdağ et al. 2011; Warner et al. 2014; Wu et al. 2014; Metivier et al. 2016), reflection waveform inversion methods (Xu et al. 2012; Alkhalifah \& Wu 2016) and geology information constrained methods (Vigh et al. 2015; Chen et al. 2016) are proposed to release requirements for initial models. For a synthetic test, the initial model usually comes from a smoothed version of the true model. Except for the cycle-skipping problem, the conventional FWI method requires many wave-equation based extrapolations to calculate the perturbations, and possibly the Hessian, and thus, incurs a huge computational cost.

There are many dimensionality-reduction approaches used to reduce the computational cost of modeling and inversion including source encoding methods (Krebs et al. 2009; BenHadj-Ali et al. 2011; Castellanos et al. 2015), randomized source methods (Moghaddam et al. 2010; van Leeuwen \& Herrmann 2013; Wang et al. 2014). Instead of reducing the extrapolation time in each iteration, for simple models, we could calculate the approximate analytical solution of the gradient based on the newly proposed impedance inversion method (Khaniani et al. 2012; Zhang \& Alkhalifah 2015, 2016; Tang \& McMechan 2016). General seismic inversion methods, including the widely used 1D impedance inversion (Oldenburg et al. 1983; Russell 1988; Yilmaz 2001; Yuan et al. 2015), provide a way to calculate velocity perturbations from the image of the data residual using perturbation theory. Under the assumption, the mild lateral velocity variation, the $1 \mathrm{D}$ inversion method is also applicable for $2 \mathrm{D}$ or $3 \mathrm{D}$ inversions. To calculate the image corresponding to velocity perturbations, migration of the data residual This article is protected by copyright. All rights reserved. 
is needed. Both wave-equation and ray-theory based depth migration methods depend vitally on the migration velocity. Actually, at the beginning of full waveform inversion, the velocity model is usually considerably inaccurate, which leads to inaccurate focusing and positioning of reflectors (perturbations). Such inaccuracies for depth based imaging tend to be larger than those corresponding to time domain imaging methods (Biondi 2006). For conventional velocity analysis, time domain processing methods, in which vertical time replaces depth as the vertical axis, are more robust (Alkhalifah 2003; Plessix 2012). The oriented time-domain imaging method developed by Fomel (2007) is independent of the velocity model and provides a better chance of guaranteeing an accurate representation of the image, especially at the beginning of the inversion process. In other words, the energy of the data is transformed to the migration image without leakage to extended images. This property is crucial for our proposed objective function which evaluates the data misfit in the image domain. Besides, local slopes estimated from prestack reflection data contain all the information of the reflection geometry (Casasanta \& Fomel 2011). Once they are calculated correctly, the seismic velocities, incident angles, and some other wave geometry parameters become data attributes. For multiparameter inversion, incident angles are needed to distinguish the contributions to the waveform from different parameters. An average velocity model is needed to convert the models from time to depth per iteration; it can be approximated better than interval velocity, and thus the proposed method provides more robust processing results than interval-velocity based method.

Previously the anisotropic parameters are not inverted in FWI; background anisotropic parameters acquired from the scanning method or well-logs are kept fixed during the waveform inversion process even for anisotropic substructures (Operto et al. 2013; Warner et al. 2013). Since the aim of FWI is to fit the full information content of observed and predicted data, the simulation engine embedded in the FWI algorithm should honor all of the physics of wave propagation as much as possible (Virieux \& Operto 2009). Multiparameter inversion including velocity, density, attenuation and anisotropic parameters helps improve the FWI results and is drawing more and more attention in full waveform inversion analysis (Plessix \& Cao 2011; Prieux et al. 2013; Alkhalifah \& Plessix 2014; Métivier et al. 2015; Wu \& Alkhalifah 2016; Kamath \& Tsvankin 2016). We propose a new multiparameter inversion method for VTI media based on the pseudo-acoustic wave equation introduced by Alkhalifah (2000). Our examples reveal the difficulties and additional requirements of multiparameter inversion in VTI media. One of the difficulties in multiparameter FWI is to eliminate the crosstalk artifacts between different parameters. One remedy is to select the optimal parametrization, which not only can adequately describe the subsurface model but also can be inverted from the observed This article is protected by copyright. All rights reserved. 
data (Plessix \& Cao 2011; Alkhalifah \& Plessix 2014; Oh et al. 2015). When considering the parameters as virtual sources in the model space, we can calculate their radiation patterns, which indicate their contributions to the waveform recorded at the surface. Their contributions depend on scattering angles in the model space or roughly offsets in the data space. When back projecting the data residual in the data domain to parameter perturbations in the model domain, the radiation patterns act like filters. Due to the limited separation of the acquisition geometry, some parameters cannot be retrieved if their unique contributions are not identified in the observed data. The parametrization analysis indicates which parameter can be retrieved reliably for a certain acquisition configuration. Surface acquisition cannot retrieve all the VTI anisotropic parameters as indicated by their radiation patterns. To be more specific, $\delta$ in VTI media cannot be inverted without prior geology information (Feng \& Schuster 2016; Xia 2016; Wu \& Alkhalifah 2016). Besides, a long-offset acquisition geometry is needed to retrieve $\eta$ from the surface recorded data (Pattnaik et al. 2016). Since far-offset data only recover the lowwavenumber part of the model, the inverted $\eta$ has lower resolution (Alkhalifah 2016). Another difficulty is the variable influence of different parameters on the waveform. The hierarchical strategies (Brossier et al. 2009; Prieux et al. 2013; Operto et al. 2013) can retrieve different parameter classes successively and partly mitigate the ill-posedness of FWI. Since incident angles help to distinguish the contributions of different parameters, the extended images in the incident angle dimension are needed. The extended images can be calculated easily because the incident angles are also data attributes.

This paper is divided into six sections. After the introduction, we propose a new objective function and derive its gradients for VTI media; as a special case, the gradient for isotropic media is given when there is no anisotropic and no angle dependence. A brief workflow explaining the implementation of our inversion is provided in section three. In the fourth section, we first test our monoparameter inversion method on the laterally smooth part of Marmousi model and then test our multiparameter inversion method on the same part of Marmousi II model and a revised Sigeesb2B model. Finally, we discuss the benefits and limitations of this method and present the summary of our work in the last section.

\section{THEORY}

\subsection{Impedance Inversion and FWI}

Impedance inversion methods provide a relationship between impedance and image in time domain (Oldenburg et al. 1983; Russell 1988). Under the assumptions that the density is This article is protected by copyright. All rights reserved. 
constant and the lateral velocity variation is negligible, the 1D inversion method can be extended to a 2D or 3D case. After converting the seismic data residual to images, we can combine the full waveform inversion method with the acoustic impedance inversion method and derive an efficient way to calculate the parameter's perturbations. In this section, we use an image domain objective function and derive the corresponding gradients for different parameters.

If we take the full waveform inversion as a least-squares local optimization problem, then the conventional misfit function is given by

$$
J_{d}=\frac{1}{2}\left\|L(m)-d^{o b s}\right\|_{2},
$$

where $m$ represents the model parameters and here in an acoustic, constant density, case, it is given by the anisotropic parameters and velocities, $L$ denotes the forward modeling operator, and $d^{o b s}$ is the observed seismic data. $J_{d}$ denotes the widely used objective function in the data domain. The operator $\|\cdot\|_{2}$ corresponds to the $l_{2}$ norm.

Instead of evaluating the data misfit directly, we calculate the misfit after converting the data residual to the time-domain image,

$$
J_{I}=\frac{1}{2}\|I(\tau, \mathbf{x})\|_{2}
$$

where $I(\tau, \mathbf{x})$ is the prestack time migration image of the data residual; $\tau$ and $\mathbf{x}$ are vertical one-way time and offset respectively. $J_{I}$ denotes the objective function we used in the image domain. Thanks to the velocity-independent property of the dip-oriented migration method, the images are always focused at the zero time lag and zero subsurface offset. In other words, equations 1 and 2 define the same global minimum because there is no energy leakage.

To minimize the new misfit function, gradient-based optimization methods are needed (Virieux \& Operto 2009). Here, we use the steepest descent method which is given by

$$
m^{i+1}=m^{i}-\alpha \frac{\partial I(\tau, \mathbf{x})}{\partial m} I(\tau, \mathbf{x})
$$

where $m$ denotes the model parameters, which could be velocity, density and anisotropic parameters according to the assumed approximations of the subsurface model, and $\alpha$ is the step length which can be calculated using a line search method (Pratt 1999). The Frechét derivation, $\frac{\partial I}{\partial m}$, has an explicit form with the help of the widely used convolutional model (Russell 1988) in conventional impedance inversion method.

The general form of the convolutional model is given by

$$
I(\tau, \mathbf{x})=R(\tau, \mathbf{x}) * s(\tau)
$$

Here, $\mathbf{x}$ denotes offset vector, $\tau$ denotes the vertical one-way traveltime. $I(\tau, \mathbf{x})$ denotes the This article is protected by copyright. All rights reserved. 
image in the time domain, $R(\tau, \mathbf{x})$ denotes the reflection coefficient and $s(\tau)$ denotes the source wavelet used for forward modeling. The symbol "*” denotes a convolution operation in this paper.

The parameters, including the velocity, density, and anisotropic parameters, are hidden in the specific form of $R(\tau, \mathbf{x})$ which depends on the assumptions made of the subsurface. In the next section, we derive the specific form of equation 3 for VTI media. As a special case, the isotropic form is also given when there is no anisotropic, and thus, no incident angle dependence.

\subsection{Multi-parameter Inversion In VTI Media}

For VTI media, the reflection coefficient given by Rüger (1997) is shown below,

$$
R_{P}^{V T I}(\theta)=\frac{1}{2} \frac{\Delta Z}{Z}+\frac{1}{2}\left(\frac{\Delta V_{p 0}}{V_{p 0}}-\left(\frac{2 V_{s 0}}{V_{p 0}}\right)^{2} \frac{\Delta G}{G}+\Delta \delta\right) \sin ^{2} \theta+\frac{1}{2}\left(\frac{\Delta V_{p 0}}{V_{p 0}}+\Delta \epsilon\right) \sin ^{2} \theta \tan ^{2} \theta,
$$

where $\theta$ denotes the incident angle, which is important to suppress the cross-talk artifacts between different parameters, $Z=\rho V_{p 0}$ is the vertical P-wave impedance, and $G=\rho V_{s 0}$ denotes the vertical shear modulus, which is assumed to be zero in our pseudo-acoustic approximation. $\Delta$ denotes the differences of the corresponding parameters between two layers. Here we set the density to equal 1. The perturbations $\Delta \delta=\delta_{2}-\delta_{1}$ and $\Delta \epsilon=\epsilon_{2}-\epsilon_{1}$ are the differences in anisotropy between two layers. Subscriptions 1 and 2 indicate the upper and lower layers respectively.

Using the approximation introduced by Russell (1988) in conventional impedance inversion,

$$
\frac{1}{2} \frac{\Delta Z(\tau, x)}{Z(\tau, x)}=\frac{1}{2} \frac{\partial \ln (Z(\tau, x))}{\partial \tau},
$$

we insert equations 6 and 5 into equation 4 and get the image $I_{P}^{V T I}(\theta)$ with regard to the pseudo-acoustic VTI reflection coefficient and the source wavelet,

$$
I_{P}^{V T I}(\theta)=\frac{1}{2}\left[\ln V_{p 0}\left(1+\sin ^{2} \theta+\sin ^{2} \theta \tan ^{2} \theta\right)\right] * \dot{s}+\frac{1}{2}\left[\Delta \delta \sin ^{2} \theta+\Delta \epsilon \sin ^{2} \theta \tan ^{2} \theta\right] * s .
$$

For inversion purpose, we apply equation 7 to the whole space domain by setting the differences of parameters equal zero within one layer. Since we're using reflection waves for inversion, only the upper layers can be updated. To utilize the small perturbation theory, the upper points of $\delta$ and $\epsilon$ are perturbed successively. Which are $\delta_{2}-\left(\delta_{1}+\delta \delta\right)$ and $\epsilon_{2}-\left(\epsilon_{1}+\delta \epsilon\right)$ respectively. The perturbed images with respect to perturbations of the parameters are given by,

$$
(I+\delta I)_{v_{p 0}}^{V T I}(\theta)=\frac{1}{2}\left[\ln \left(V_{p 0}+\delta V_{p 0}\right)\left(1+\sin ^{2} \theta+\sin ^{2} \theta \tan ^{2} \theta\right)\right] * \dot{s}
$$




$$
\begin{gathered}
+\frac{1}{2}\left[\left(\delta_{2}-\delta_{1}\right) \sin ^{2} \theta+\left(\epsilon_{2}-\epsilon_{1}\right) \sin ^{2} \theta \tan ^{2} \theta\right] * s, \\
(I+\delta I)_{\delta}^{V T I}(\theta)=\frac{1}{2}\left[\ln V_{p 0}\left(1+\sin ^{2} \theta+\sin ^{2} \theta \tan ^{2} \theta\right)\right] * \dot{s} \\
+\frac{1}{2}\left[\left(\delta_{2}-\left(\delta_{1}+\delta \delta\right)\right) \sin ^{2} \theta+\left(\epsilon_{2}-\epsilon_{1}\right) \sin ^{2} \theta \tan ^{2} \theta\right] * s, \\
(I+\delta I)_{\epsilon}^{V T I}(\theta)=\frac{1}{2}\left[\ln V_{p 0}\left(1+\sin ^{2} \theta+\sin ^{2} \theta \tan ^{2} \theta\right)\right] * \dot{s} \\
+\frac{1}{2}\left[\left(\delta_{2}-\delta_{1}\right) \sin ^{2} \theta+\left(\epsilon_{2}-\left(\epsilon_{1}+\delta \epsilon\right)\right) \sin ^{2} \theta \tan ^{2} \theta\right] * s .
\end{gathered}
$$

Here $\delta V_{p 0}, \delta \delta$ and $\delta \epsilon$ denote perturbations of $V_{p 0}, \delta$ and $\epsilon$ respectively. The potential perturbations exist anywhere in the space except the last layer, which means the last layer cannot be updated. This is consistent with the property of the conventional reflection waveform inversion method. The last layer cannot be updated if there are no reflections from the bottom of the model.

Subtracting equation 7 from equation 8 and using Taylor expansion for $\ln (1+x) \approx x$, when $x$ is small, we get the relationships between perturbed parameters and the image of data residual, given by

$$
\begin{gathered}
\delta I_{v_{p 0}}^{V T I}(\theta) \approx \frac{1}{2}\left[\frac{\delta V_{p 0}}{V_{p 0}}\left(1+\sin ^{2} \theta+\sin ^{2} \theta \tan ^{2} \theta\right)\right] * \dot{s} \\
\delta I_{\delta}^{V T I}(\theta) \approx \frac{1}{2}\left[\delta \delta \sin ^{2} \theta\right] * \tilde{s} \\
\delta I_{\epsilon}^{V T I}(\theta) \approx \frac{1}{2}\left[\delta \epsilon \sin ^{2} \theta \tan ^{2} \theta\right] * \tilde{s} .
\end{gathered}
$$

To be consistent, we use the polarity flipped source wavelet $\tilde{s}$ to generate the perturbed image. By virtue of the associativity law of convolution and integral over the reflection angles, the gradients with respect to the proposed objective function $J_{I}$ are given by

$$
\begin{gathered}
\nabla_{J_{I}}^{v_{p 0}} \approx \frac{1}{2}\left[\frac{\sum_{\theta} I(\tau, \mathbf{X}, \theta)\left(1+\sin ^{2} \theta+\sin ^{2} \theta \tan ^{2} \theta\right)}{V_{p 0}}\right] * \dot{s}, \\
\nabla_{J_{I}}^{\delta} \approx \frac{1}{2}\left[\sum_{\theta} I(\tau, \mathbf{x}, \theta) \sin ^{2} \theta\right] * \tilde{s}, \\
\nabla_{J_{I}}^{\epsilon} \approx \frac{1}{2}\left[\sum_{\theta} I(\tau, \mathbf{x}, \theta) \sin ^{2} \theta \tan ^{2} \theta\right] * \tilde{s},
\end{gathered}
$$


where $I(\tau, \mathbf{x}, \theta)$ is the extended image along the incident angle axis. The incident angle exposes the varying contributions to the waveform from the parameters, $V_{p 0}, \delta$ and $\epsilon$, which is used to mitigate the cross-talk artifacts between them in the inversion step. In addition to utilizing the incident angle directly, we also want to choose an optimal parameter set which has fewer overlaps as a function of incident angle. More details about choosing the optimal parametrization for our proposed method are shown in the next section.

The derivation for monoparameter case is straightforward. Note that equation 5 is a good approximation for the reflection coefficient of vertical-incident $\mathrm{P}$-wave by setting the shear velocity, anisotropic parameters and incident angle equal zero. Thus, the reduced form of equation 10 is the gradient for the monoparameter inversion such that the vertical $\mathrm{P}$-wave velocity inversion is given by,

$$
\nabla_{J_{I}}^{v_{p 0}} \approx \frac{1}{2}\left[\frac{I(\tau, \mathbf{x})}{V_{p 0}}\right] * \dot{s} .
$$

No incident angle information is needed since we only invert for a monoparameter, vertical P-wave velocity. At last, the steepest descent method could be implemented to minimize the proposed objective function.

\subsection{Parametrization}

For multiparameter inversion, the coupled effects of the parameters prevent the convergence (Operto et al. 2013; Alkhalifah \& Plessix 2014). Usually, different parameters have different sensitivities to different scattering angles, which are also called radiation patterns. In this part, we test two sets of parameters based on the previous analysis (i.e. equation 9) and choose the optimal one based on our inversion purpose and inversion strategy. Figure 1 shows the radiation patterns for the first set which includes $V_{p 0}, \delta$, and $\epsilon$. It is generated from equation 9 after normalization. It is obvious that $V_{p 0}$ is sensitive to all the scattering angles. On the other hand, $\delta$ and $\epsilon$ have overlapped scattering angles, which means that they have some cross-talk errors if we invert these two parameters simultaneously. This set of parameters is reasonable if we keep $\delta$ and $\epsilon$ fixed and invert $V_{p 0}$ only. However, if we want to invert all three parameters simultaneously, we need to change the parametrization. By virtue of the chain rule, we represent the VTI media by the following parameters $V_{n}, \eta$ and $\delta$.

$$
\left|\begin{array}{c}
\frac{\partial I}{\partial V_{n}} \\
\frac{\partial I}{\partial \eta} \\
\frac{\partial I}{\partial \delta}
\end{array}\right|=\left|\begin{array}{ccc}
\frac{\partial V_{p 0}}{\partial V_{n}} & \frac{\partial \epsilon}{\partial V_{n}} & \frac{\partial \delta}{\partial V_{n}} \\
\frac{\partial V_{p 0}}{\partial \eta} & \frac{\partial \epsilon}{\partial \eta} & \frac{\partial \delta}{\partial \eta} \\
\frac{\partial V_{p 0}}{\partial \delta} & \frac{\partial \epsilon}{\partial \delta} & \frac{\partial \delta}{\partial \delta}
\end{array}\right|\left|\begin{array}{c}
\frac{\partial I}{\partial V_{p 0}} \\
\frac{\partial I}{\partial \epsilon} \\
\frac{\partial I}{\partial \delta}
\end{array}\right| .
$$

The relationships between different anisotropic parameters are written as, This article is protected by copyright. All rights reserved. 


$$
\begin{array}{ll}
V_{n}=V_{p 0} \sqrt{1+2 \delta} & \begin{array}{l}
V_{p 0}=\frac{V_{n}}{\sqrt{1+2 \delta}} \\
\eta=\frac{\epsilon-\delta}{1+2 \delta}
\end{array} \quad \begin{array}{l}
\epsilon=(1+2 \delta) \eta+\delta . \\
\delta=\delta
\end{array}
\end{array}
$$

Here $\delta$ is taken as an independent parameter. Then we can get a new formula for the parameter perturbations and the image of the data residual, given by

$$
\left|\begin{array}{c}
\frac{\delta I}{\delta V_{n}} \\
\frac{\delta I}{\delta \eta} \\
\frac{\delta I}{\delta \delta}
\end{array}\right| \approx\left|\begin{array}{ccc}
\frac{1}{\sqrt{1+2 \delta}} & 0 & 0 \\
0 & 1+2 \delta & 0 \\
-V_{n}(1+2 \delta)^{-\frac{3}{2}} & 1+2 \eta & 1
\end{array}\right|\left|\begin{array}{c}
\frac{\delta I}{\delta V_{p 0}} \\
\frac{\delta I}{\delta \epsilon} \\
\frac{\delta I}{\delta \delta}
\end{array}\right| .
$$

The gradients for $V_{n}, \eta$ and $\delta$ are given by,

$$
\left|\begin{array}{c}
\nabla_{J_{I}}^{v_{n}} \\
\nabla_{J_{I}}^{\eta} \\
\nabla_{J_{I}}^{\delta}
\end{array}\right| \approx\left|\begin{array}{ccc}
\frac{1}{\sqrt{1+2 \delta}} & 0 & 0 \\
0 & 1+2 \delta & 0 \\
-V_{n}(1+2 \delta)^{-\frac{3}{2}} & 1+2 \eta & 1
\end{array}\right|\left|\begin{array}{c}
\nabla_{J_{I}}^{v_{p 0}} \\
\nabla_{J_{I}}^{\epsilon} \\
\nabla_{J_{I}}^{\delta}
\end{array}\right| .
$$

The radiation patterns for parameters $V_{n}, \eta$ and $\delta$ are shown in Figure 2. Similar to Figure 1, it is generated from equation 15. For this set of parameters, $V_{n}$ is sensitive to all the scattering angles, $\delta$ is more sensitive to small scattering angles and $\eta$ is more sensitive to large scattering angles. Thus, we choose this set of parameters as the optimal one since we intend to recover all the parameters of the subsurface. The cross-talk effects between $\delta$ and $\eta$ could be eliminated by adding the scattering angle information in the inversion process. To reduce the variable effects of $V_{n}$ and the other two parameters, we invert $V_{n}$ for the first several iterations and then invert these three parameters jointly, which is referred to as the hierarchy method (Brossier et al. 2009; Prieux et al. 2013) and is fully described in our workflow.

\subsection{Extended Oriented Time-domain Imaging Method}

The oriented time-domain imaging method developed by Fomel (2007) applies mainly a prestack time migration utilizing velocity information extracted from the local event slopes in the reflection data. Thus, this method is independent of the velocity model, which could help us avoid putting the reflectors (or perturbations) in the wrong position due to the erroneous initial velocity model. This method is applicable for VTI media under the assumption that the moveout is non-hyperbolic. Here, we only share the basic idea behind the oriented time-domain imaging method, and for more details we refer you to Fomel (2007).

The basic idea of this migration method is to find a mapping relationship between the time-domain image and data attributes. The time-migrated image domain $(\tau, x)$ is mappable from the prestack domain $(t, h, y)$ using the following formulas, This article is protected by copyright. All rights reserved. 


$$
\tau^{2}=\frac{t p_{h}\left[\left(t-h p_{h}\right)^{2}-h^{2} p_{y}^{2}\right]^{2}}{\left(t-h p_{h}\right)^{2}\left[t p_{h}+h\left(p_{y}^{2}-p_{h}^{2}\right)\right]}
$$

$$
x=y-\frac{h t p_{y}}{t p_{h}+h\left(p_{y}^{2}-p_{h}^{2}\right)},
$$

where $h$ denotes the half-offset, $x$ denotes the midpoint in the image domain and $y$ denotes the midpoint in the data domain. The event slopes in recorded data, given by $p_{h}=\partial t / \partial h$ and $p_{y}=\partial t / \partial y$, can be estimated using the plane-wave destruction algorithm of Fomel (2002). Different from the isotropic case, we need to calculate the subsurface incident angle using equation 18 besides the vertical one-way time $\tau$ and midpoint $x$ in the image domain for the anisotropic case. Since the incident angle is one of the attributes of the data, the extended images can be easily calculated by sweeping over all the possible subsurface incident angles and reorganize them in the incident angle dimension.

$$
\sin ^{2} \theta=\frac{h p_{h}}{t}
$$

This equation will be used to generate extended images in our proposed multiparameter inversion.

\section{THE WORKFLOW FOR THIS METHOD}

In summary, the hierarchy-inversion strategy used for the multiparameter inversion includes the following steps:

(i) Establish initial parameter models in time. The initial guesses should be accurate enough to avoid ending up in a local minimum.

(ii) Select a frequency band to invert within the data.

(iii) Converting current models from time to depth using average vertical velocity $v_{p 0}$, calculate the synthetic data by solving the pseudo-acoustic wave equation for VTI media, which was first introduced by Alkhalifah (2000). $v_{p 0}$ is updated by $v_{p 0}=\frac{v_{n}}{\sqrt{1+2 \delta}}$ in each iteration; $v_{n}$ and $\delta$ are inverted parameters per iteration.

(iv) Evaluate the data residual for each shot and organize them into CMP gathers.

(v) Migrate the data residual using the oriented time-domain imaging method. Here we calculate the extended images along the incident-angle axis.

(vi) Calculate the gradients in the time domain.

(vii) Update $V_{n}$ for the first several iterations and then parameters $\delta$ and $\eta$ using a multipoint line search method.

This article is protected by copyright. All rights reserved. 
(viii) Repeat steps (iii)-(vii) until the data residual is not decreasing.

(ix) Repeat steps (ii)-(viii) for the multi-scale approach until the data residual is not decreasing and the frequency range from low to high is covered (i.e. 0-4, 0-6, 0-8 and 0-10 $\mathrm{Hz})$.

For monoparameter inversion, the implementation is more straightforward and simple, we need to solve the pure acoustic wave equation to generate the data in step (iii), and only $\mathrm{P}$-wave velocity is inverted in step (vii). The only significant computational cost in this implementation is generating the observed data by solving the wave equation.

\section{NUMERICAL EXAMPLES}

\subsection{Monoparameter Case}

We use the multi-scale method introduced by Bunks et al. (1995) for the inversion and start with a peak frequency of $4 \mathrm{~Hz}$. There is a total of four frequency selection stages used here including 4, 6, 8 and $10 \mathrm{~Hz}$, and each stage includes five iterations. Each simulation has an absorbing boundary condition and is free of direct arrivals. According to the assumptions of our approach, we choose the left, laterally smooth, part of the Marmousi velocity model to test our method. The model size is 400 by 500 grids with a grid interval of $4 \mathrm{~m}$. We consider 50 shots with 100 geophones in each shot uniformly distributed in the horizontal direction. Each shot is a point-source with known Ricker wavelet.

The initial velocity shown in Figure $3 \mathrm{~b}$ is a smoothed version of the true velocity model shown in Figure 3a. The smoothing operator is given by a triangle filtering with a window size of $1 / 4$ of the total grid points in depth and $1 / 10$ of the total grid points in the horizontal direction. Figure $3 \mathrm{c}$ shows the inverted velocity model. To make the comparison more intuitive, we plot the velocities versus depth in Figure 4. There are no updates for the shallow part because the reflections from the shallow part are removed with the direct wave. For the deeper part, the initial velocity has inaccurate kinematic information and thus the inverted one is not good. As limited-bandwidth data is used for inversion, not all the wavenumber components can be recovered. Thus, we also plot the wavenumber filtered true velocity for comparison (Alkhalifah 2016). The inverted velocity is close to the filtered true velocity in the middle part mainly because the initial velocity contains accurate wavenumber components in that part. However, in the deep part where a large velocity jump exists, the initial velocity is far from the true velocity and thus the proposed method can not provide a good result. Figure 5 illustrates the normalized data residual for the first stage. The other stages share This article is protected by copyright. All rights reserved. 
similar decreasing curves since only the peak frequency of the source changes from one stage to another. The data residual decreases as a function of iteration steps, which means that the simulated data fits the observed one better and better per iteration.

The computational cost of the time migration method used in our example is negligible comparing to the computational cost of shot-by-shot wavefield extrapolations. The comparison depends on the approach used in the conventional FWI method. If the conventional FWI method is implemented by boundary reconstruction strategy, the proposed method saves about two-thirds of the total computational cost per iteration. If the whole wavefield is kept in the memory then the proposed method saves about half of the total computational cost per iteration. Besides, the proposed method do not require saving or reconstructing the wavefields and thus has a small memory cost.

\subsection{Multiparameter Case}

We solve the pseudo-acoustic wave equation proposed by Alkhalifah (2000) using the finitedifference method in our multiparameter inversion examples. Both examples share the same size of the model which is 400 by 500 grids with a space sampling of $6.096 \mathrm{~m}$. We consider 50 shots with 100 geophones in each shot uniformly distributed in the horizontal direction. Each shot is a point-source with exact source wavelet.

The first example is part of revised Sigsbee2B model. In conformity with our assumptions, we select the horizontal part of the original model and reproduce anisotropic parameters through the velocity model. The inversion starts with a peak frequency of $2 \mathrm{~Hz}$ and end with $5 \mathrm{~Hz}$, totally 4 stages with an interval of $1 \mathrm{~Hz}$. There are 15 iterations per stage and only $V_{n}$ is updated at the first 8 iterations. The depth of water layer is assumed to be known.

Figure 6 shows the initial models used for multiparameter inversion, they're smoothed version of the true models shown in Figures $7 \mathrm{a}, 7 \mathrm{~b}$ and $7 \mathrm{c}$, respectively. The inverted $V_{n}, \eta$ and $\delta$ are shown in Figures $7 \mathrm{~d}, 7 \mathrm{e}$ and $7 \mathrm{f}$, respectively. There are no updates for the shallow part mainly because the reflections are removed as first arrivals. The updates for $\eta$ are small compared to $V_{n}$ and $\delta$ because of the limited offset to depth ratio. As a quality control factor, we also plot the vertical profiles in Figure 8. For $V_{n}$ and $\delta$, most layers can be recovered correctly, but there are some crosstalk artifacts between them. $\delta$ might be helpful in fitting the amplitudes even they cannot be recovered correctly. The inverted $\eta$ has lower resolution than the other two parameters because only large offset data contributes to the inverted $\eta$, which is the low wavenumber part. The results are consistent with the previous analysis on radiation patterns. As an important quality control parameter, a curve of normalized data This article is protected by copyright. All rights reserved. 
misfit versus iterations for the 1st stage is plotted in Figure 9. It shows that the anisotropic parameters help reduce the data misfit further. Although there are inherent crosstalks between $V_{n}$ and $\delta, \delta$ acts as a "garbage" parameter and helps fit the amplitude which can not be done by the velocity itself.

The next synthetic example is part of Marmousi II model, and the original model is not anisotropic. We use the following equations $\epsilon=0.25 \rho-0.3$ and $\delta=0.125 \rho-0.1$ to generate the anisotropic models (Yan \& Sava 2009). We also use the multi-scale method for the inversion and begin with a peak frequency of $2 \mathrm{~Hz}$. There is a total of 4 frequency selection stages used here with a range of 2, 3, 4 and $5 \mathrm{~Hz}$, and each stage contains twenty iterations. Only the parameter $V_{n}$ is updated in the first ten iterations in each stage, then $V_{n}, \eta$ and $\delta$ are inverted simultaneously. The water layer is set to be isotropic, and the velocity is assumed to be already known.

The initial models shown in Figure 10 are smoothed versions of the true models shown in Figures 11a, 11b, and 11c. The smoothing operator is given by a triangle filtering with a window size of $1 / 10$ of the total grid points in depth and $1 / 10$ of the total grid points in the horizontal direction. Figures 11d, 11e, and 11f are inverted velocity, inverted $\eta$ and inverted $\delta$ at $5 \mathrm{~Hz}$, respectively. The updating range of $\eta$ in Figure 11e decreases with increasing depth because $\eta$ contributes more to the far offset data, which matches the previous analysis of parametrization. To make the comparison more intuitive, we plot the parameters versus depth at $x=1.5 \mathrm{~km}$ in Figure 12. There are no updates for the shallow part because the reflections are removed with first arrivals. The $V_{n}$ profile shown in Figure 12a can be well recovered since it has an isotropic radiation pattern, thus influencing the full offset range. We only managed to invert the shallow part of $\eta$ shown in Figure 12b. Since the maximum peak frequency used in the inversion is $5 \mathrm{~Hz}$, the resolution of the inverted parameter $\delta$ is not very high. More details of the models are expected to be retrieved if we use higher peak frequencies in the inversion. Besides, there are still some cross talks between $V_{n}$ and $\delta$ as some updates for $\delta$ is not correct. These artifacts can not be removed because there is an overlap between $V_{n}$ and $\delta$ as shown in Figure 2. Similarly, the normalized data misfit versus iterations of the 1st stage plotted in Figure 13 shows that although the remaining data residual is large, anisotropic parameters help fit the data better. To further reduce the trade-offs and therefore the data residual, we suggest to use approximate Hessian in the inversion process. This article is protected by copyright. All rights reserved. 


\section{DISCUSSIONS}

Considering the inaccurate velocities we tend to work with at the beginning of the FWI process, the gradient is usually far from perfect. To be more specific, imperfect initial models not only generate the so-called local minima problem but also provide inaccurate Green's function in the backpropagation step when using the adjoint-state method for FWI. Although the proposed method needs accurate kinematic information to mitigate the local minima problem, its gradient is independent of the models once the data residual is given. An average velocity model is needed for time to depth conversion in order to generate the predicted data. From the prestack and poststack migration practice, the average velocity is more easily estimated than the interval velocity. The proposed approach is based on the assumptions that the density of the medium is constant, and the lateral velocity change is small. Subject to the prestack time-domain migration limitations, the velocity model can not be too complicated. The cycle skipping problem is a troublesome problem for local optimization methods and to avoid it we will need a kinematic-accurate initial velocity model. The numerical examples indicate that the performance of this method depends on the accuracy of the initial velocity model to obtain proper residual. The results are stable overall thanks to the time domain implementation. Time-domain based imaging tends to be less sensitive to inaccurate velocities than depth ones. The new formula for calculating the velocity perturbation is also derived as a special case when there is no anisotropic and no angle dependence. From the radiation patterns of the anisotropic parameters, we can find the data dependency on different parameters. In the data domain, $V_{n}$ has equal contributions to all scattering angles, and thus, influences all offsets, which allows for a reasonable retrieval of it from the data. On the other hand, $\delta$ mainly affects the amplitude of the near-offset data, and thus, it promotes the waveform fitting at short offsets in FWI. Meanwhile, $\eta$ mainly affects the traveltime of far-offset data. Thus, the updating capability of $\eta$ reduces with depth in the proposed method.

The real value of this approach is in its ability to handle elastic or anisotropy in a more robust matter. The opportunity to divide the update process to an imaging step (mapping the data residual to model space) and a parameter inversion step (using the analytical Frechét derivation to calculate the gradient) allows for better control of the anisotropy update. A featured seismic impedance inversion method has been utilized for years. However, we utilize this at the update stage maintaining the more elaborate (FWI) objective of fitting the modeled data to the observed ones. This approach promises to deliver a practical orthorhombic 
16

much larger (double at minimum), but so is the required azimuth coverage in the acquisition to hopefully constrain these parameters.

\section{CONCLUSIONS}

We propose a new objective function in time image domain based on the fact that the energy of migration image of the data residual should be zero when the predicted data and observed data are the same. Thanks to the oriented time domain imaging method, the migration image always focus at zero subsurface offset. Thus, no extended migration images are needed to evaluate the data misfit in the image domain. Besides, using the oriented time-domain migration method allows us to avoid relying on the initial velocity model for the gradient once the data residual is given and thus provides more stable updates. Although a time to depth conversion is needed to generate the predicted data per iteration. Considering the poor velocity models we tend to start FWI with, the velocity free gradient calculation can help improve convergence as its objective has a more convex nature. After time-domain imaging, approximated analytical Frechét derivatives are applied to map the image of data residual to the corresponding perturbations of model parameters. Thus, the proposed method has less computational cost than the adjoint-state method with a cost of an approximated gradient. The proposed method has some assumptions to the subsurface models, however, it should be valid for common layered models in practice.

This method is applicable to VTI anisotropic media. By extending the oriented timedomain migration image along incident angle axis, we can separate the data dependency on different anisotropic parameters, which helps eliminate the crosstalk artifacts between them. We also analyze different sets of anisotropic parameters based on the proposed method and choose an optimal parametrization for our proposed VTI anisotropic inversion method, and the numerical examples verify its effectiveness.

\section{ACKNOWLEDGMENTS}

We thank the editors and two anonymous reviewers for constructive suggestions that improved the manuscript greatly. We thank KAUST for its support and specifically the seismic wave analysis group members for their valuable insights. We thank Ehsan Naeini from Ikon sciences for useful discussions. Zhen-dong Zhang thanks Yike Liu for his help and also the National Nature Science Foundation of China (Grant No. 41430321) for its funding.

This article is protected by copyright. All rights reserved. 


\section{REFERENCES}

Alkhalifah, T., 2000. An acoustic wave equation for anisotropic media, Geophysics, 65(4), 1239-1250.

Alkhalifah, T., 2003. Tau migration and velocity analysis: Theory and synthetic examples, Geophysics, 68(4), 1331-1339.

Alkhalifah, T., 2016. Full-model wavenumber inversion: An emphasis on the appropriate wavenumber continuation, Geophysics, 81(3), R89-R98.

Alkhalifah, T. \& Plessix, R.-É., 2014. A recipe for practical full-waveform inversion in anisotropic media: An analytical parameter resolution study, Geophysics, 79(3), R91-R101.

Alkhalifah, T. \& Wu, Z., 2016. The natural combination of full and image-based waveform inversion, Geophysical Prospecting, 64(1), 19-30.

Ben-Hadj-Ali, H., Operto, S., \& Virieux, J., 2011. An efficient frequency-domain full waveform inversion method using simultaneous encoded sources, Geophysics, 76(4), R109-R124.

Biondi, B., 2006. 3D seismic imaging, Society of Exploration Geophysicists Tulsa, Okla, USA.

Bozdağ, E., Trampert, J., \& Tromp, J., 2011. Misfit functions for full waveform inversion based on instantaneous phase and envelope measurements, Geophysical Journal International, 185(2), 845-870.

Brossier, R., Operto, S., Virieux, J., et al., 2009. Two-dimensional seismic imaging of the valhall model from synthetic obc data by frequency domain elastic full-waveform inversion, in 2009 SEG Annual Meeting, Society of Exploration Geophysicists.

Bunks, C., Saleck, F. M., Zaleski, S., \& Chavent, G., 1995. Multiscale seismic waveform inversion, Geophysics, 60(5), 1457-1473.

Casasanta, L. \& Fomel, S., 2011. Velocity-independent $\tau$-p moveout in a horizontally layered vti medium, Geophysics, 76(4), U45-U57.

Castellanos, C., Métivier, L., Operto, S., Brossier, R., \& Virieux, J., 2015. Fast full waveform inversion with source encoding and second-order optimization methods, Geophysical Journal International, $\mathbf{2 0 0}(2), 718-742$.

Chen, Y., Chen, H., Xiang, K., \& Chen, X., 2016. Geological structure guided well log interpolation for high-fidelity full waveform inversion, Geophysical Journal International, 207(2), 1313-1331.

Datta, D., Sen, M., Liu, F., \& Morton, S., 2016. Salt model building by shape-based parameterization and global fwi, in SEG Technical Program Expanded Abstracts 2016, pp. 1069-1073, Society of Exploration Geophysicists.

Feng, S. \& Schuster, G., 2016. Anisotropic wave-equation traveltime and waveform inversion, in SEG Technical Program Expanded Abstracts 2016, pp. 1196-1200, Society of Exploration Geophysicists. Fomel, S., 2002. Applications of plane-wave destruction filters, Geophysics, 67(6), 1946-1960.

Fomel, S., 2007. Velocity-independent time-domain seismic imaging using local event slopes, Geophysics, 72(3), S139-S147.

Kamath, N. \& Tsvankin, I., 2016. Elastic full-waveform inversion for vti media: Methodology and This article is protected by copyright. All rights reserved. 
sensitivity analysis, Geophysics, 81(2), C53-C68.

Khaniani, H., John, B., \& Gary, M., 2012. Full waveform inversion algorithm using time imaging methods, in CSPG/CSEG/CWLS GeoConvention 2012.

Krebs, J. R., Anderson, J. E., Hinkley, D., Neelamani, R., Lee, S., Baumstein, A., \& Lacasse, M.-D., 2009. Fast full-wavefield seismic inversion using encoded sources, Geophysics, 74(6), WCC177WCC188.

Lailly, P., 1983. The seismic inverse problem as a sequence of before stack migrations, in Conference on inverse scattering: theory and application, pp. 206-220, Society for Industrial and Applied Mathematics, Philadelphia, PA.

Liu, Y., Sun, H., \& Chang, X., 2010. Fast velocity analysis by wave path migration, Geophysical Prospecting, 58(2), 175-190.

Luo, Y. \& Schuster, G. T., 1991. Wave-equation traveltime inversion, Geophysics, 56(5), 645-653.

Métivier, L., Brossier, R., Operto, S., \& Virieux, J., 2015. Acoustic multi-parameter fwi for the reconstruction of p-wave velocity, density and attenuation: preconditioned truncated newton approach, in 2015 SEG Annual Meeting, Society of Exploration Geophysicists.

Metivier, L., Brossier, R., Oudet, E., Mérigot, Q., \& Virieux, J., 2016. An optimal transport distance for full-waveform inversion: Application to the 2014 chevron benchmark data set, in SEG Technical Program Expanded Abstracts 2016, pp. 1278-1283, Society of Exploration Geophysicists.

Moghaddam, P. P., Herrmann, F. J., et al., 2010. Randomized full-waveform inversion: a dimenstionality-reduction approach, in 2010 SEG Annual Meeting, Society of Exploration Geophysicists.

Oh, J.-W., Alkhalifah, T., \& Min, D.-J., 2015. Multi-stage full waveform inversion strategy for 2d elastic vti media, in SEG Technical Program Expanded Abstracts 2015, pp. 1204-1208, Society of Exploration Geophysicists.

Oldenburg, D., Scheuer, T., \& Levy, S., 1983. Recovery of the acoustic impedance from reflection seismograms, Geophysics, 48(10), 1318-1337.

Operto, S., Gholami, Y., Prieux, V., Ribodetti, A., Brossier, R., Metivier, L., \& Virieux, J., 2013. A guided tour of multiparameter full-waveform inversion with multicomponent data: From theory to practice, The Leading Edge, 32(9), 1040-1054.

Pattnaik, S., Tsvankin, I., Wang, H., \& Alkhalifah, T., 2016. Full-waveform inversion with reflected waves for 2d vti media, in SEG Technical Program Expanded Abstracts 2016, pp. 413-418, Society of Exploration Geophysicists.

Plessix, R.-É., 2012. A pseudo-time formulation for acoustic full waveform inversion, Geophysical Journal International, p. ggs056.

Plessix, R.-É. \& Cao, Q., 2011. A parametrization study for surface seismic full waveform inversion in an acoustic vertical transversely isotropic medium, Geophysical Journal International, 185(1), 539-556.

This article is protected by copyright. All rights reserved. 
Pratt, R. G., 1999. Seismic waveform inversion in the frequency domain, part 1: Theory and verification in a physical scale model, Geophysics, 64(3), 888-901.

Prieux, V., Brossier, R., Operto, S., \& Virieux, J., 2013. Multiparameter full waveform inversion of multicomponent ocean-bottom-cable data from the valhall field. part 1: imaging compressional wave speed, density and attenuation, Geophysical Journal International, p. ggt177.

Rüger, A., 1997. P-wave reflection coefficients for transversely isotropic models with vertical and horizontal axis of symmetry, Geophysics, 62(3), 713-722.

Russell, B. H., 1988. Introduction to seismic inversion methods, Society of Exploration Geophysicists. Sen, M. K. \& Stoffa, P. L., 1991. Nonlinear one-dimensional seismic waveform inversion using simulated annealing, Geophysics, 56(10), 1624-1638.

Tang, C. \& McMechan, G., 2016. From classical reflectivity-to-velocity inversion to full-waveform inversion using phase-modified and deconvolved reverse-time-migration images, in SEG Technical Program Expanded Abstracts 2016, pp. 1141-1145, Society of Exploration Geophysicists.

Tarantola, A., 1984. Inversion of seismic reflection data in the acoustic approximation, Geophysics, 49(8), 1259-1266.

van Leeuwen, T. \& Herrmann, F. J., 2013. Fast waveform inversion without source-encoding, Geophysical Prospecting, 61(s1), 10-19.

Van Leeuwen, T. \& Mulder, W., 2010. A correlation-based misfit criterion for wave-equation traveltime tomography, Geophysical Journal International, 182(3), 1383-1394.

Vigh, D., Lewis, W., Parekh, C., Jiao, K., \& Kapoor, J., 2015. Well constraints in full waveform inversion and its application in time-lapse seismic, in 77th EAGE Conference and Exhibition 2015.

Virieux, J. \& Operto, S., 2009. An overview of full-waveform inversion in exploration geophysics, Geophysics, 74(6), WCC1-WCC26.

Wang, C., Yingst, D., Brittan, J., Farmer, P., \& Leveille, J., 2014. Fast multi-parameter anisotropic full waveform inversion with irregular shot sampling, in SEG Technical Program Expanded Abstracts 2014, pp. 1147-1151, Society of Exploration Geophysicists.

Warner, M., Ratcliffe, A., Nangoo, T., Morgan, J., Umpleby, A., Shah, N., Vinje, V., Štekl, I., Guasch, L., Win, C., et al., 2013. Anisotropic 3d full-waveform inversion, Geophysics, 78(2), R59-R80.

Warner, M., Guasch, L., et al., 2014. Adaptive waveform inversion: Theory, in 2014 SEG Annual Meeting, Society of Exploration Geophysicists.

Weibull, W., Arntsen, B., Nilsen, E., et al., 2012. Initial velocity models for full waveform inversion, in 2012 SEG Annual Meeting, Society of Exploration Geophysicists.

Wu, R.-S., Luo, J., \& Wu, B., 2014. Seismic envelope inversion and modulation signal model, Geophysics, 79(3), WA13-WA24.

Wu, Z. \& Alkhalifah, T., 2016. Waveform inversion for acoustic vti media in frequency domain, in $S E G$ Technical Program Expanded Abstracts 2016, pp. 1184-1189, Society of Exploration Geophysicists. Xia, F., 2016. Semblance-based anisotropy parameter estimation using isotropic depth-migrated 
common image gathers, in SEG Technical Program Expanded Abstracts 2016, pp. 285-290, Society of Exploration Geophysicists.

Xu, S., Wang, D., Chen, F., Zhang, Y., \& Lambare, G., 2012. Full waveform inversion for reflected seismic data, in 74th EAGE Conference and Exhibition incorporating EUROPEC 2012.

Yan, J. \& Sava, P., 2009. Elastic wave-mode separation for vti media, Geophysics, 74(5), WB19WB32.

Yilmaz, Ö., 2001. Seismic data analysis, Society of exploration geophysicists Tulsa.

Yuan, S., Wang, S., Luo, C., \& He, Y., 2015. Simultaneous multitrace impedance inversion with transform-domain sparsity promotion, Geophysics, 80(2), R71-R80.

Zhang, Z. \& Alkhalifah, T., 2016. Full waveform inversion using oriented time-domain imaging method for vti medium, in 78th EAGE Conference and Exhibition 2016.

Zhang, Z.-D. \& Alkhalifah, T., 2015. Full waveform inversion using oriented time-domain imaging method, in 2015 SEG Annual Meeting, Society of Exploration Geophysicists. 

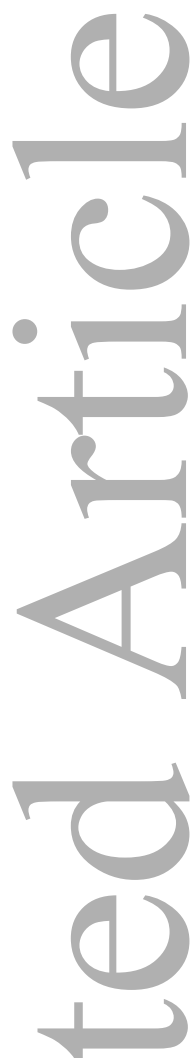

Figure 1. Radiation pattern for parameters $V_{p 0}, \delta$ and $\epsilon$. The red line indicates $V_{p 0}$; the green line indicates $\epsilon$, and the blue line indicates $\delta$. This plot is generated from equation 9 . 


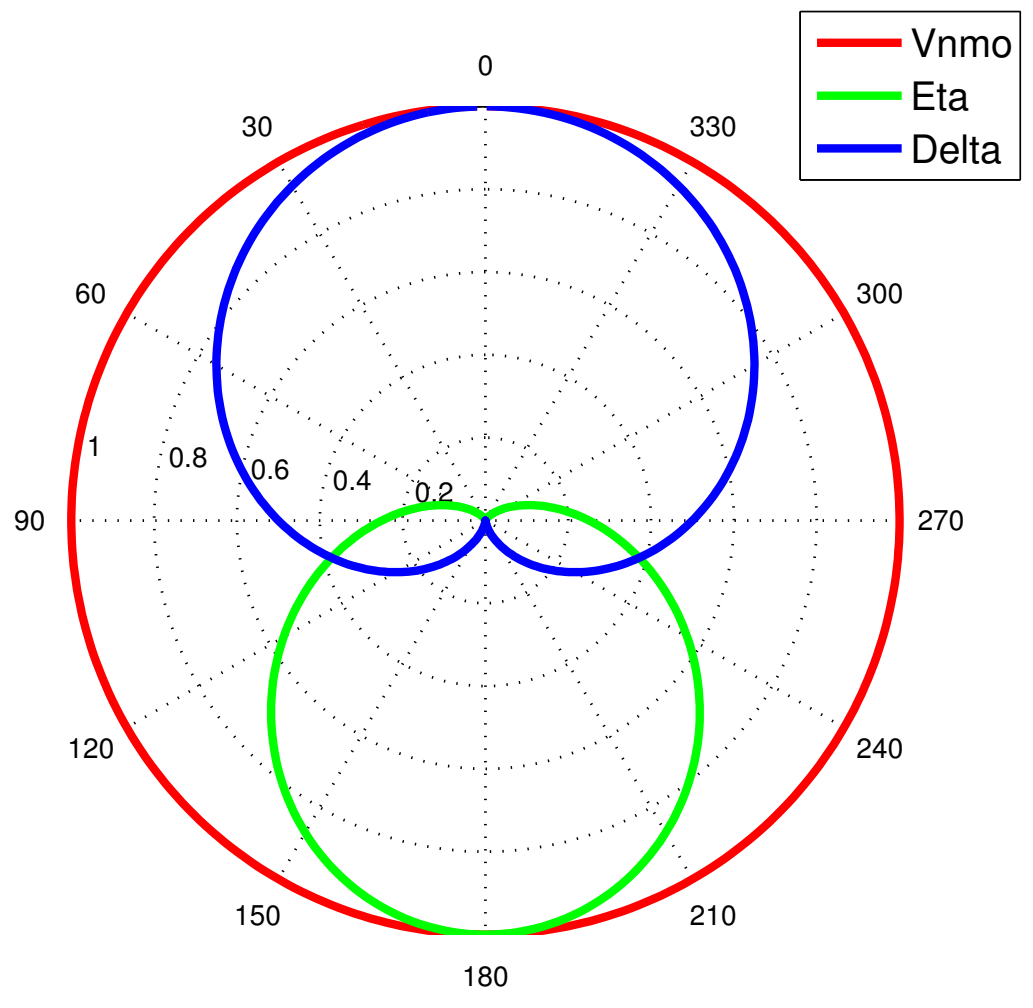

Figure 2. Radiation pattern for parameters $V_{n}, \eta$ and $\delta$. The red line indicates $V_{n}$; the green line indicates $\eta$, and the blue line indicates $\delta$. 

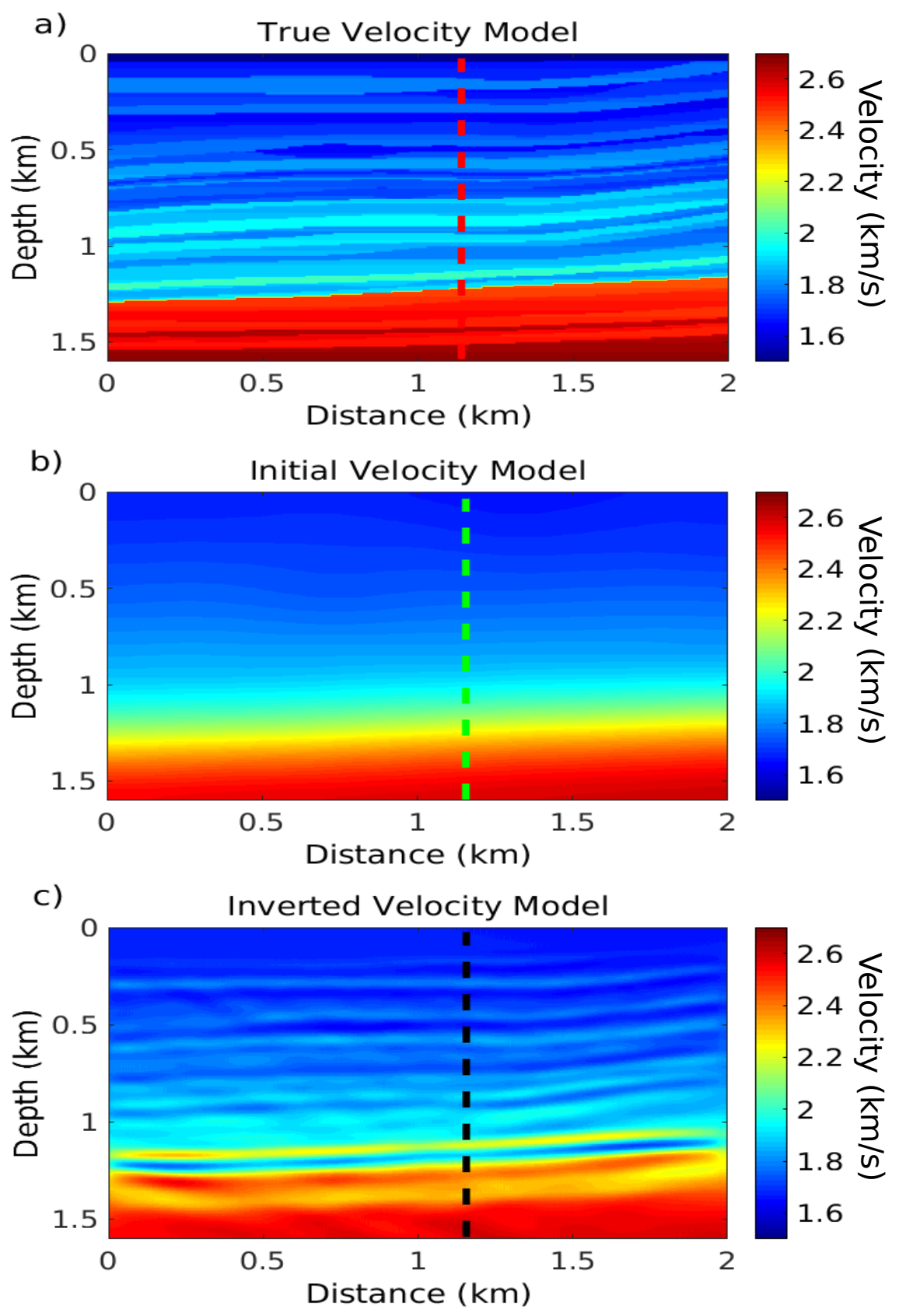

Figure 3. Monoparameter inversion results: (a) true velocity model, (b) initial velocity model, and (c) inverted velocity model. 


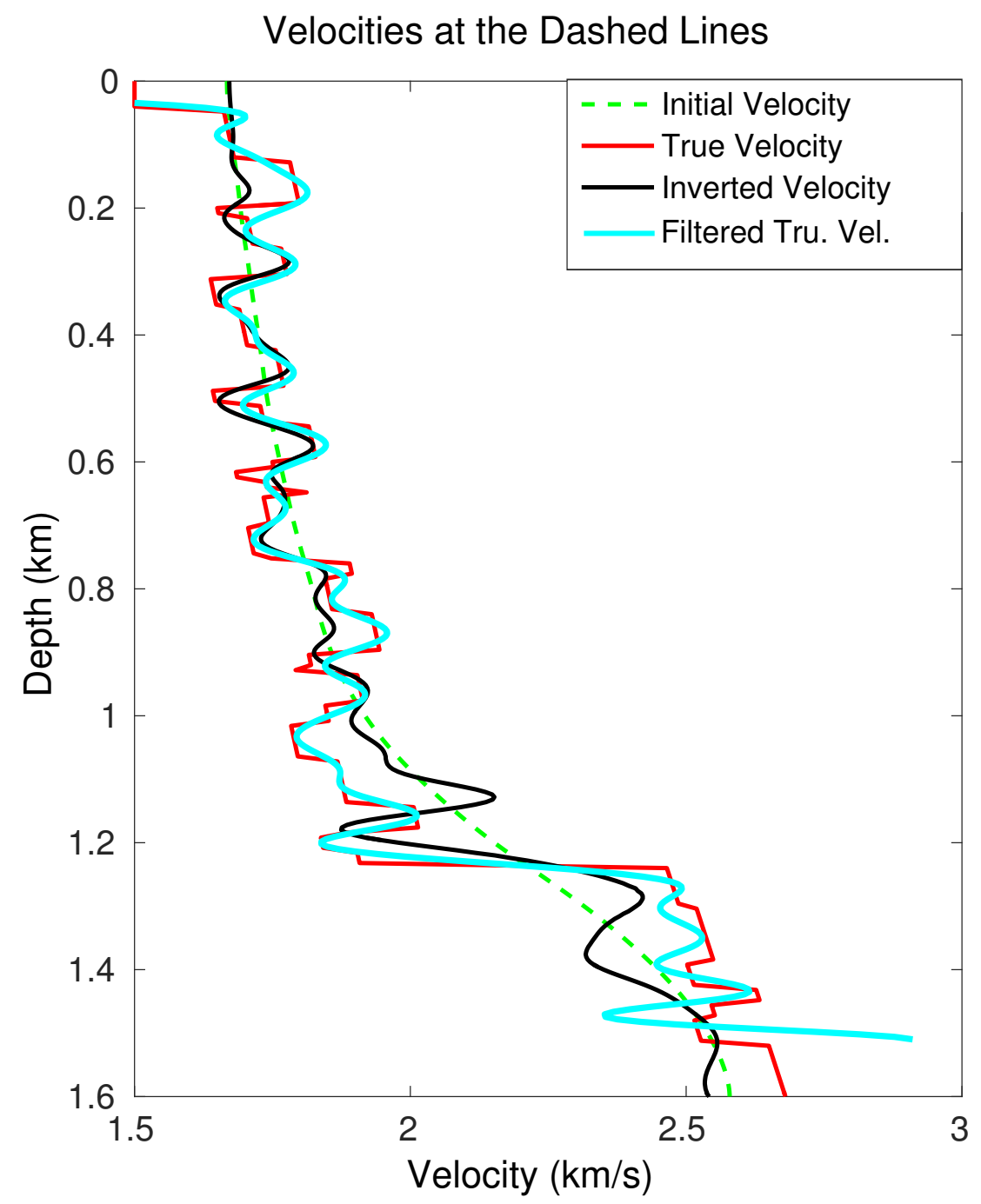

Figure 4. Comparison of velocity models. Red and cyan lines indicate true and wavnumber-filtered velocities, respectively; the dashed green line indicates initial velocity, and the black line indicates inverted velocity. 


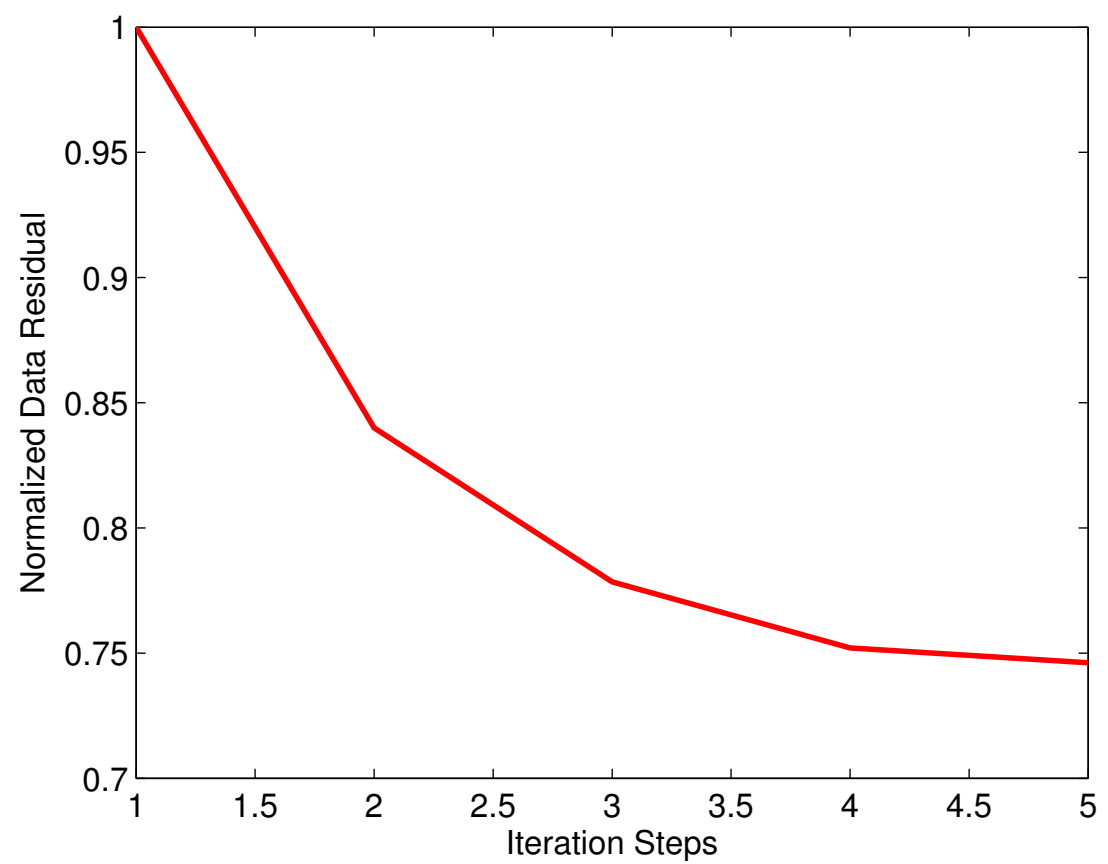

Figure 5. Normalized misfit for five iterations at $4 \mathrm{~Hz}$. The data residual is decreasing with the iteration steps increasing. More iterations should be implemented to get a better approximation to the true velocity in one stage. 


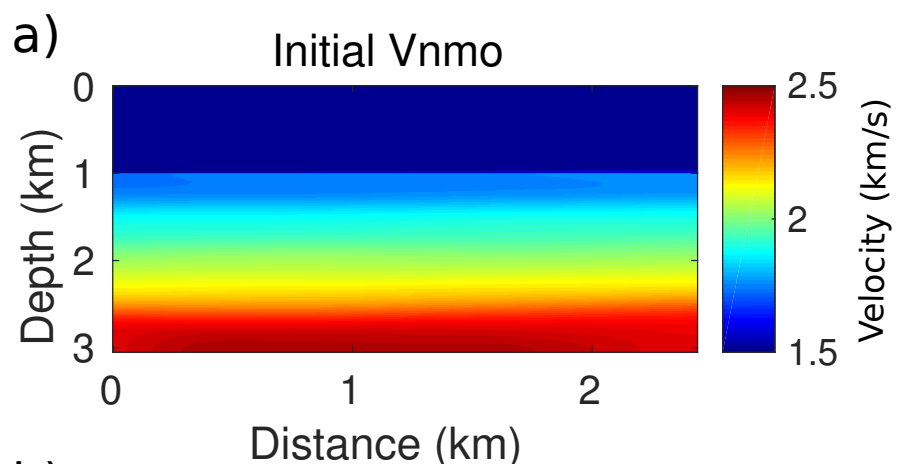

b) Initial Eta

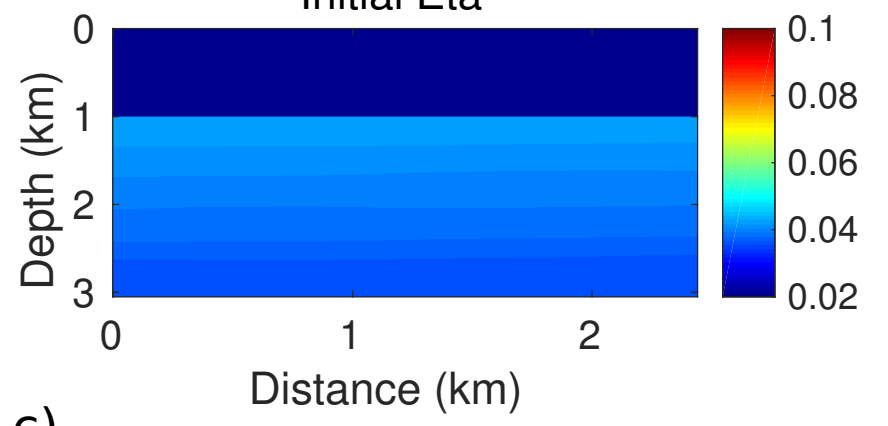

C) Initial Delta

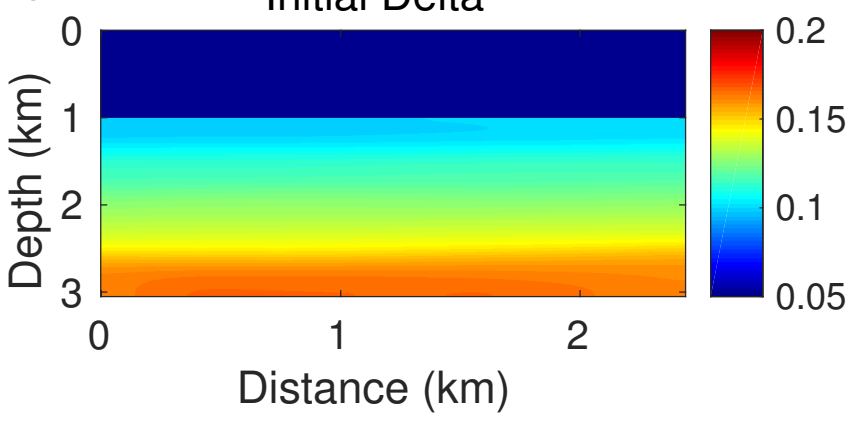

Figure 6. Initial models used in the first mulitiparameter inversion: (a) $V_{n}$, (b) $\eta$ and (c) $\delta$. They are smoothed version of the true models. 

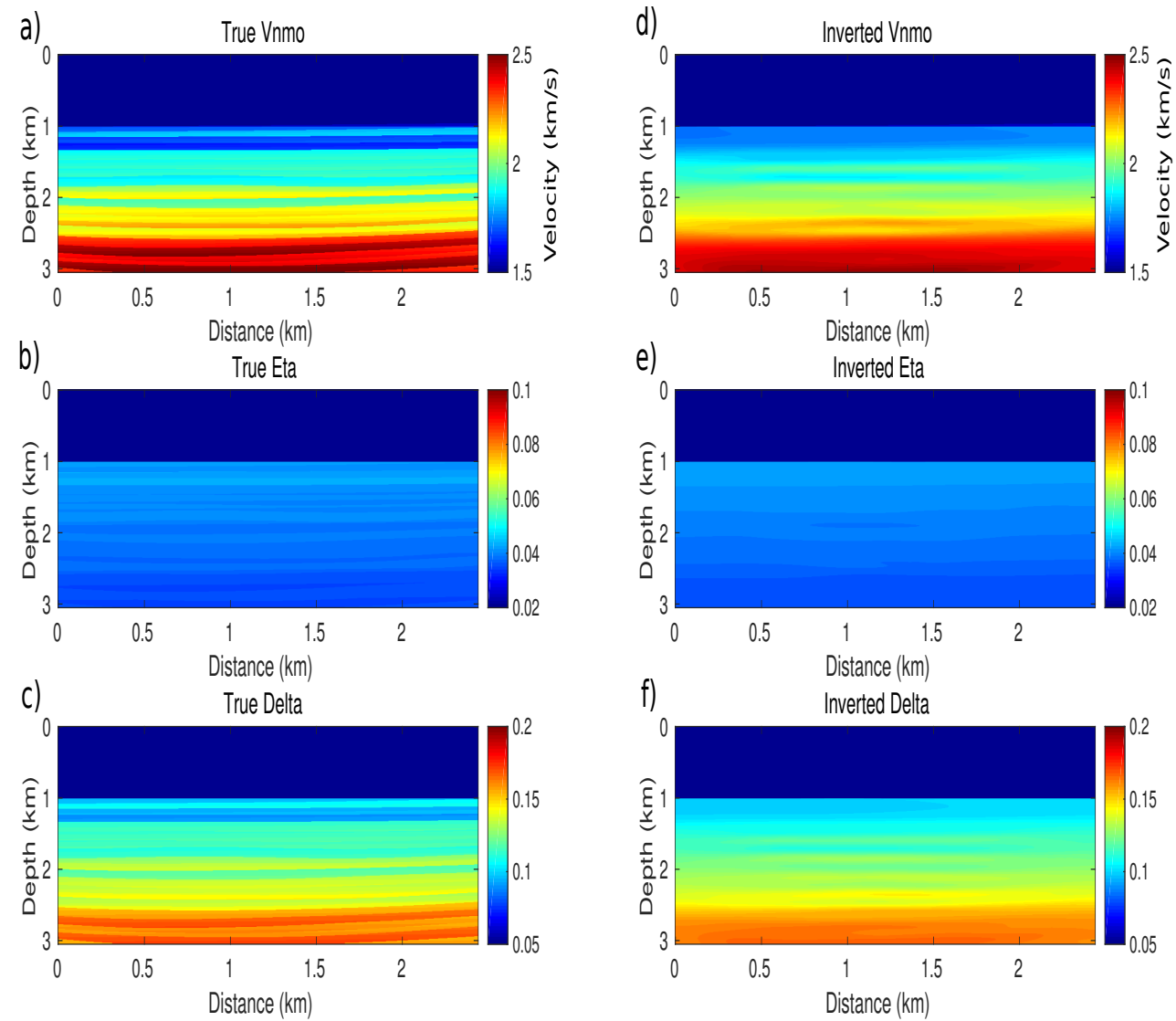

Figure 7. Multiparameter inversion results: (a) true $V_{n}$ model, (b) true $\eta$ model, (c) true $\delta$ model, (d) inverted $V_{n}$ model, (e) inverted $\eta$ model and (f) inverted $\delta$ model. 

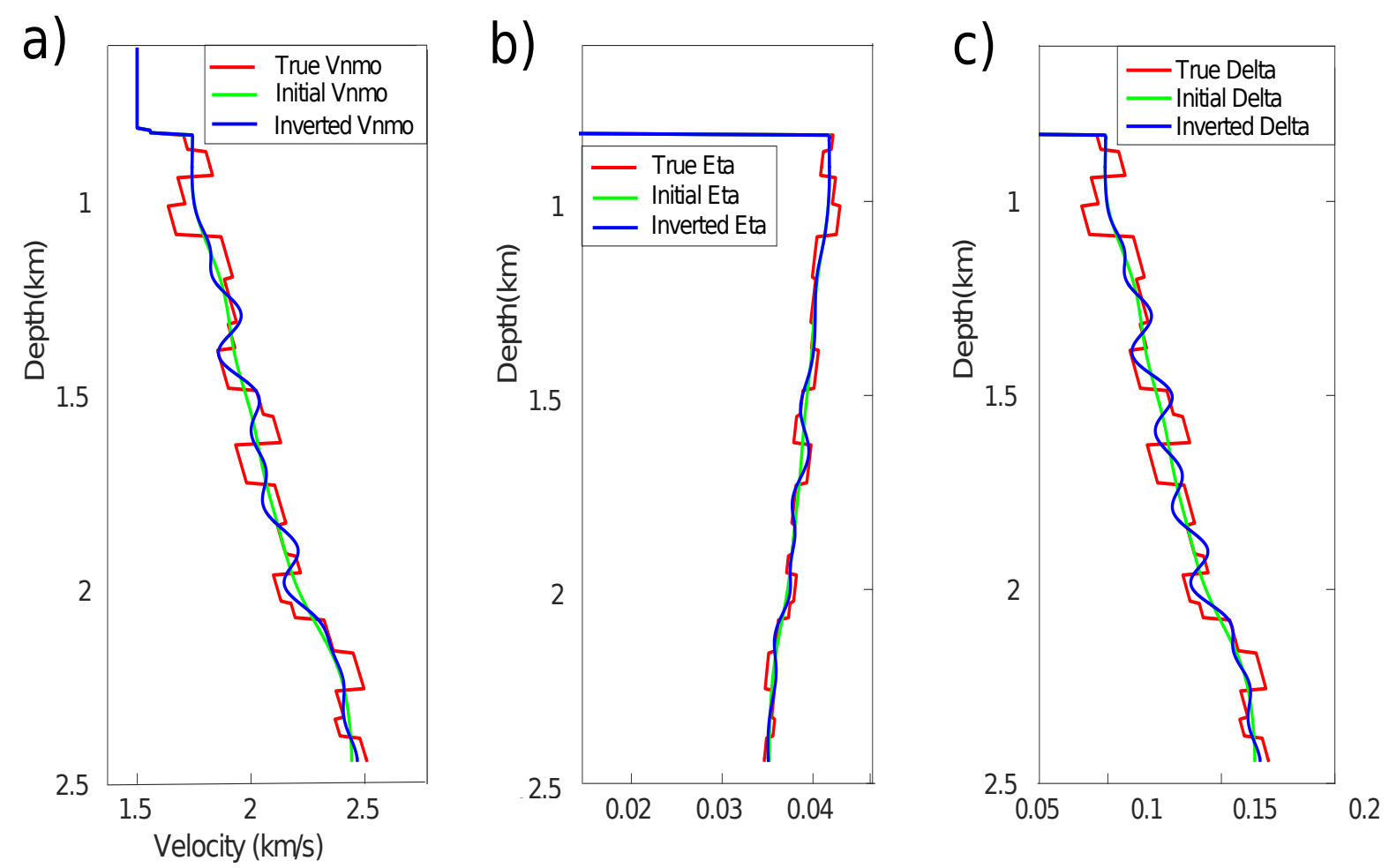

Figure 8. Comparison of the inverted models at $x=1.5 \mathrm{~km}$. (a) The red line indicates true $V_{n}$; the green line indicates initial $V_{n}$, and the blue line indicates the inverted $V_{n}$. (b) The red line indicates true $\eta$; the green line indicates initial $\eta$, and the blue line indicates the inverted $\eta$. (c) The red line indicates true $\delta$; the green line indicates initial $\delta$, and the blue line indicates the inverted $\delta$. 


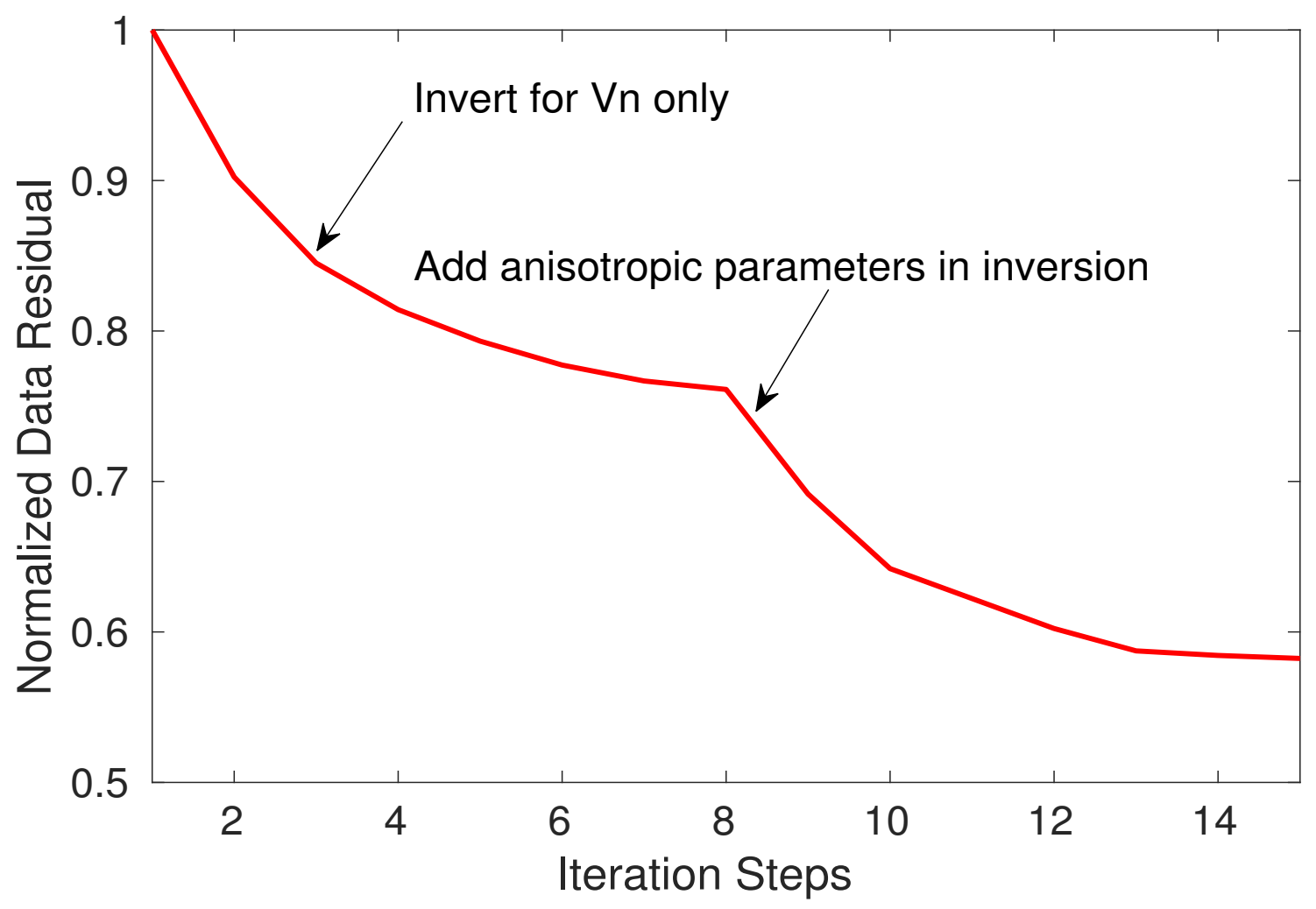

Figure 9. Normalized data misfit versus iterations for $0-4 \mathrm{~Hz}$. Only $V_{n}$ is inverted in the first 8 iterations, thereafter $V_{n}, \eta$ and $\delta$ are inverted simultaneously (from the 9th iteration). Anisotropic parameters help reduce the data misfit further, mainly because $\delta$ helps fit the amplitudes although $V_{n}$ and $\delta$ have inherent crosstalks. $\delta$ can be taken as a "garbage" parameter which helps fit the data. 

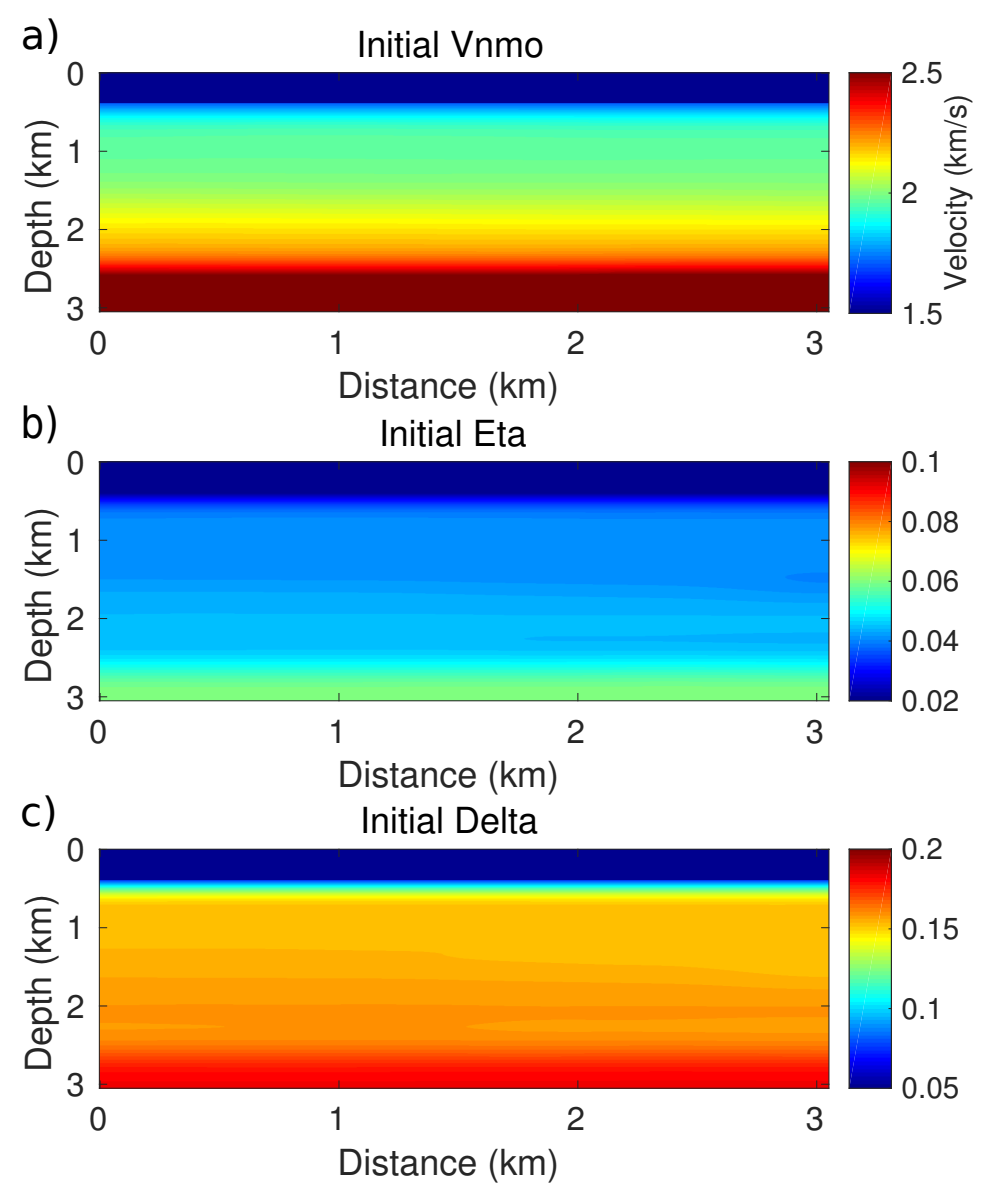

Figure 10. Initial models used in the second mulitiparameter inversion: (a) $V_{n}$, (b) $\eta$ and (c) $\delta$. They are smoothed version of the true models. 

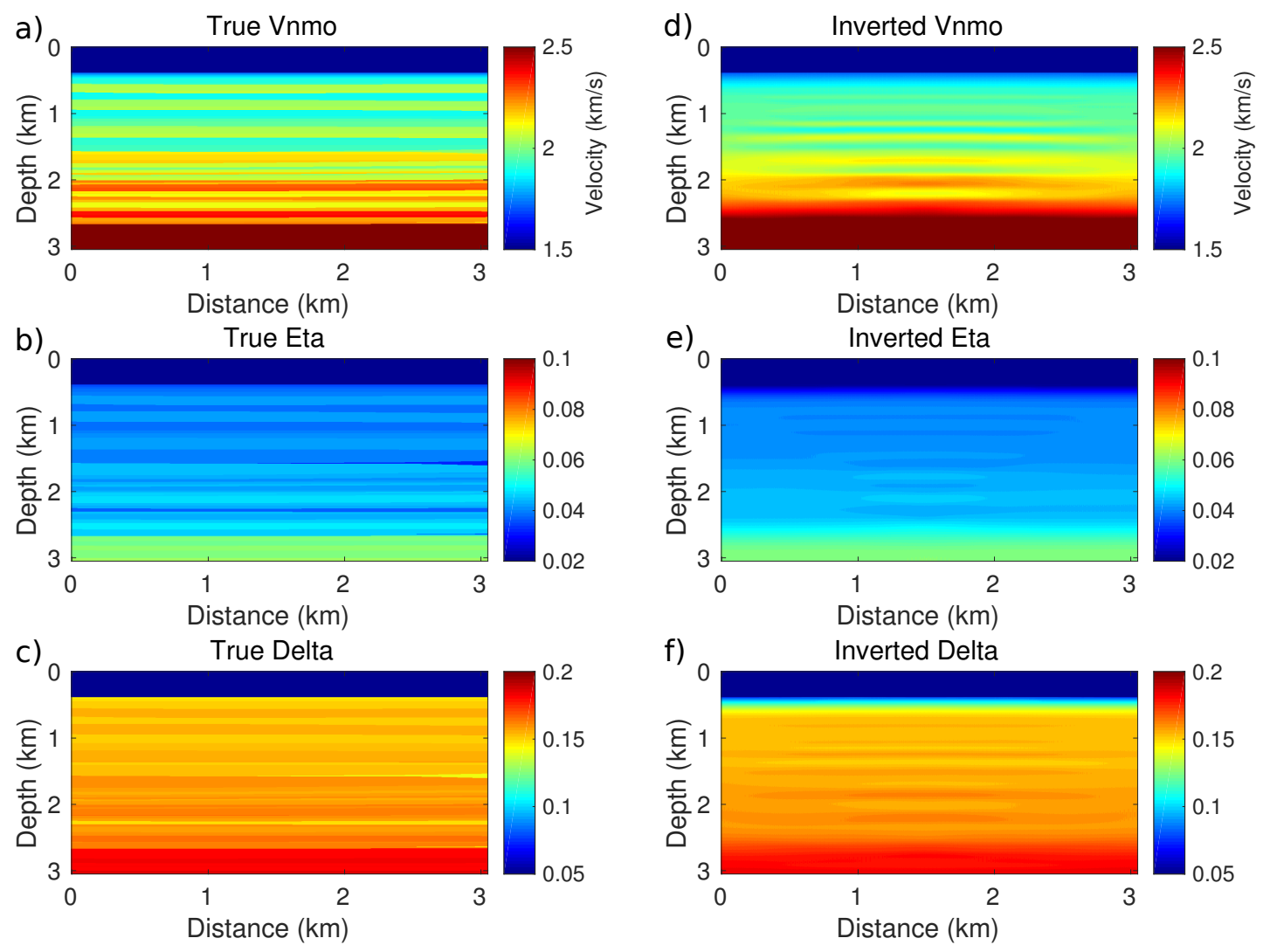

Figure 11. Multiparameter inversion results: (a) true $V_{n}$ model, (b) true $\eta$ model, (c) true $\delta$ model, (d) inverted $V_{n}$ model, (e) inverted $\eta$ model and (f) inverted $\delta$ model. 

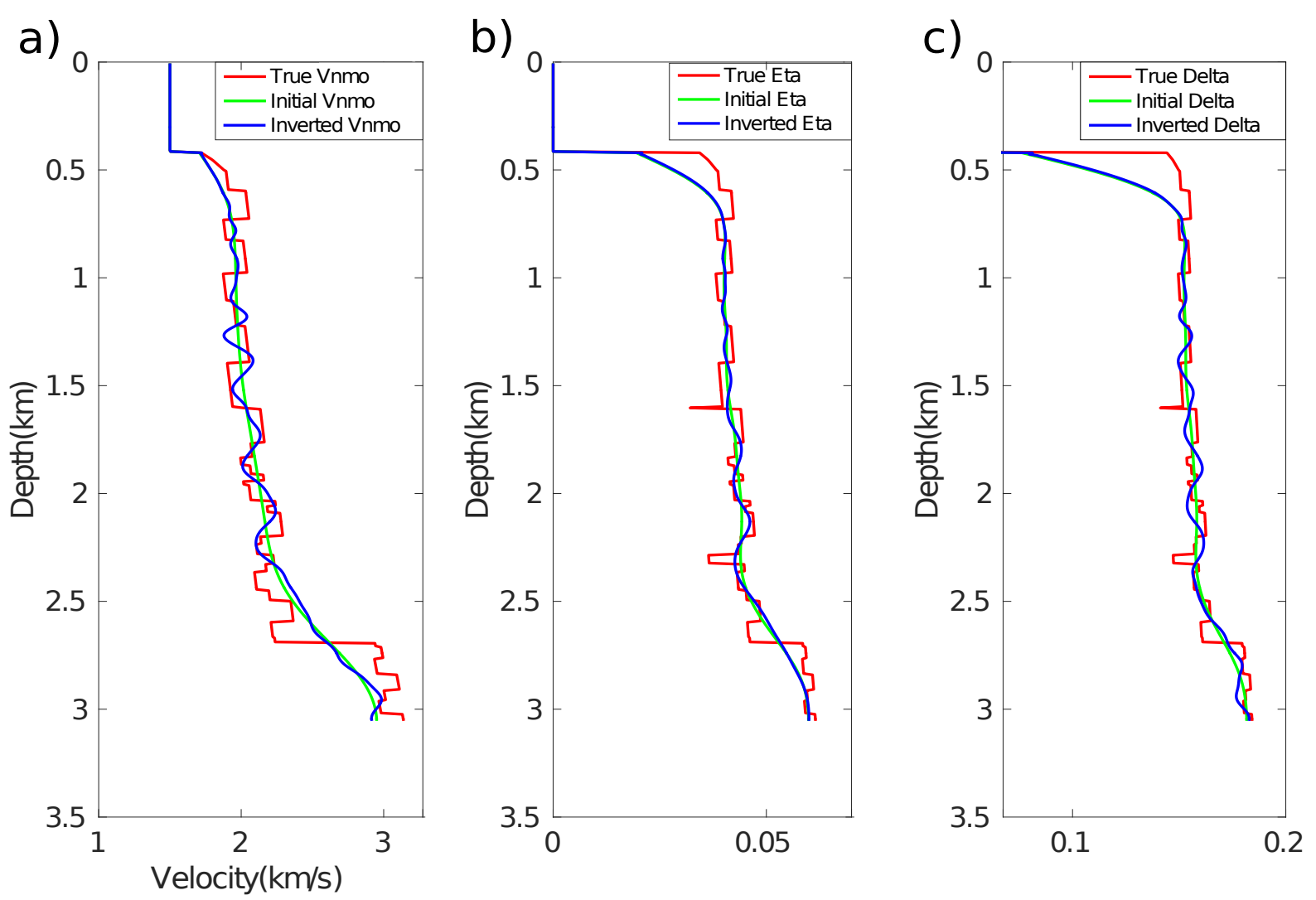

Figure 12. Comparison of the inverted models at $x=1.5 \mathrm{~km}$. (a) The red line indicates true $V_{n}$; the green line indicates initial $V_{n}$, and the blue line indicates the inverted $V_{n}$. (b) The red line indicates true $\eta$; the green line indicates initial $\eta$, and the blue line indicates the inverted $\eta$. (c) The red line indicates true $\delta$; the green line indicates initial $\delta$, and the blue line indicates the inverted $\delta$. 


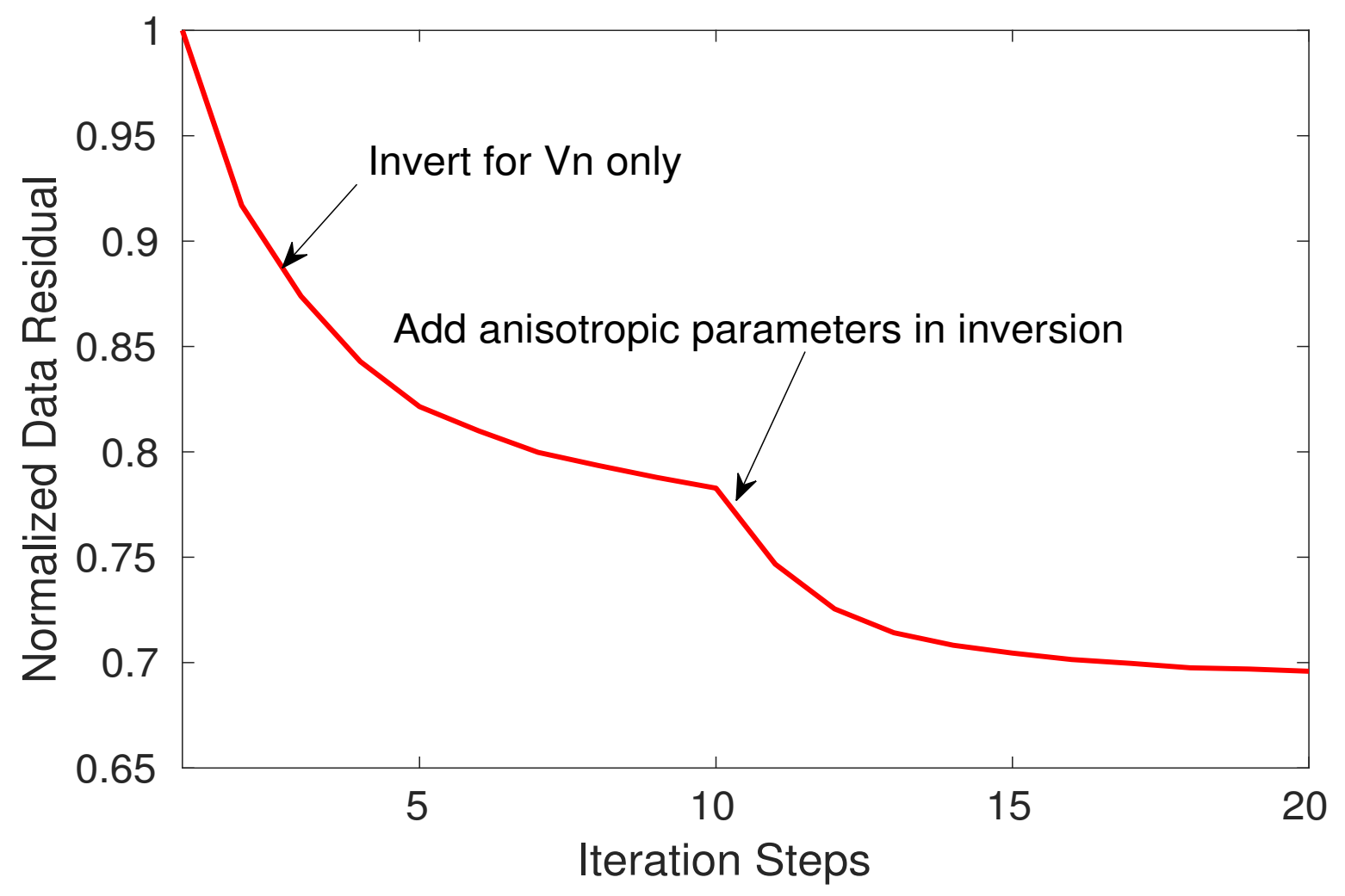

Figure 13. Normalized data misfit versus iterations for $0-4 \mathrm{~Hz}$. Only $V_{n}$ is inverted in the first 10 iterations, thereafter $V_{n}, \eta$ and $\delta$ are inverted simultaneously (from the 11st iteration). 